\title{
ACADEME-LOCAL GOVERNMENT PARTNERSHIP TOWARDS EFFECTIVE APPLICATION OF GEOSPATIAL TECHNOLOGIES FOR SMARTER FLOOD DISASTER MANAGEMENT AT THE LOCAL LEVEL: AN EXAMPLE FROM MINDANAO, PHILIPPINES
}

\author{
M. Makinano-Santillan, ${ }^{\mathrm{a}, \mathrm{b} *}$ J. R. Santillan ${ }^{\mathrm{a}}$, E. M. O. Morales ${ }^{\mathrm{a}}$, L. C. S. Asube ${ }^{\mathrm{a}, \mathrm{b}}$, A. M. Amora ${ }^{\mathrm{a}}$, L. C. Cutamora ${ }^{\mathrm{a}}$, R. M. Makinano ${ }^{\mathrm{a}}$ \\ ${ }^{a}$ CSU Phil-LiDAR 1 Project, Caraga Center for Geo-Informatics \\ College of Engineering and Information Technology, Caraga State University \\ Ampayon, Butuan City, Agusan del Norte, Philippines - mmsantillan@ carsu.edu.ph \\ ${ }^{\mathrm{b}}$ Division of Geodetic Engineering \\ College of Engineering and Information Technology, Caraga State University \\ Ampayon, Butuan City, Agusan del Norte, Philippines
}

Commission VIII, WG VIII/1

KEY WORDS: Geospatial technology, Flood, Disaster management, Academe-local government partnership, Mindanao, Philippines

\begin{abstract}
:
In this paper, we discuss how an academe-local government partnership can lead the way for the effective use of geospatial technologies for smarter and geospatially-informed decision making before, during, and after a flood disaster. In Jabonga municipality, in the province of Agusan del Norte, in Mindanao, Philippines, two significant flooding events occurred in the year 2014 which were caused by overflowing water bodies due to continuous heavy rains. These flood events inundated populated areas, caused massive evacuation, made roads un-passable, and greatly damaged sources of incomes such as croplands and other agricultural areas. The partnership between Caraga State University and the local government of Jabonga attempts to improve localized flood disaster management through the development of web-based Near-real Time Flood Event Visualization and Damage Estimations (Flood EViDEns) application. Flood EViDENs utilizes LiDAR-derived elevation and information products as well as other elevation datasets, water level records by monitoring stations, flood simulation models, flood hazard maps, and socio-economic datasets (population, household information, etc.), in order to visualize in near-real time the current and future extent of flooding, to disseminate early warnings, and to provide maps and statistics of areas and communities affected and to be affected by flooding. The development of Flood EViDEns as the main product of the partnership is an important application of geospatial technologies that will allow smarter and geospatially-informed decision making before, during, and after a flood disaster in Jabonga.
\end{abstract}

\section{INTRODUCTION}

Flood-related disasters in the Philippines have become more pronounced in recent years, majority of which have been caused by tropical storms and low pressure systems which bring along rains of varying duration, volume and intensity. In Jabonga municipality, in the province of Agusan del Norte, in Mindanao, Philippines, two significant flooding events occurred in the year 2014 (one in January, and another in December), which were mainly caused by the overflowing of several water bodies, particularly Lake Mainit, due to continuous heavy rains. These flood events inundated populated areas, caused massive evacuation, made roads un-passable, and greatly damaged sources of incomes such as croplands and other agricultural areas. Preparations and management actions exercised by the Local Government Units (LGUs) for these flood disasters b are already considered standard operating procedures in accordance with the Philippines' National Disaster Risk Reduction and Management Plan (NDRRMP) 2011-2028 (NDRRMC, 2011) and in the National Disaster Response Plan (DRRM-CEP, 2014). However, some interventions in the procedures are essential to improve localized flood disaster management. These interventions call for timely, efficient and systematic dissemination of early warnings to communities; for determining before-hand sets of information necessary for careful planning and preparation of evacuation strategies;

\footnotetext{
${ }^{*}$ Corresponding author
}

for easily identifying areas that need immediate action; in identifying areas that should be avoided; and in estimating the severity of damage to people and infrastructure as flooding progresses.

In this paper, we discuss how these proposed interventions were made possible using geospatial technologies through a partnership between the academe (CSU Phil-LiDAR 1 Project of Caraga State University) and the Jabonga LGU. The objective of this partnership is to pave the way for effective use of geospatial technologies for smarter and geospatially-informed decision making before, during, and after a flood disaster in the Jabonga municipality through the development a web-based application called "Near-real Time Flood Event Visualization and Damage Estimations (Flood EViDEns)".

\section{THE CSU PHIL-LIDAR 1 PROJECT}

The CSU Phil-LiDAR 1 is one of several projects under the "PhilLiDAR1: Hazard Mapping of the Philippines using LiDAR" program initiated and supported by the Department of Science and Technology (DOST). Projects under this program are being implemented nationwide by fifteen (15) higher education institutions (HEIs) for 3 years since 2014, and its primary aim is to generate flood hazard maps through the use of high spatial resolution Light Detection and Ranging (LiDAR) and other geospatial datasets together and techniques. The CSU Phil-LiDAR 1, in particular, started in April 2014 and covers 12 rivers basins in Caraga 
Region, Mindanao, which includes Jabonga, among many other cities and municipalities (Figure 1). Details about the project are discussed in Makinano-Santillan and Santillan (2015). However, we provide here some salient features of the project, especially on how the project generates 1-m spatial resolution hazard maps of covered river basins.

Within the CSU Phil-LIDAR 1 project, the steps involved in flood hazard map generation are streamlined into 3 major activities namely, (i) field surveying, (ii) LiDAR data processing, and (iii) flood modelling. All field data collection activities of the project are being handled by the Field Survey Team (FST). Gathering information from the field ensures that the actual characteristics of the rivers and its floodplains are captured. Knowing how much rain falls into the watershed, how wide and deep the river is, how fast the water flows, and how much water flows along the river would allow us to determine the capacity of the river and what levels it would take for the river to overflow. To gather all this information, the FST conducts river cross-section surveys and flow measurements through installation of rain gauges, water level sensors, and velocity meters.

LiDAR datasets of the rivers systems covered by the project are being processed by the Data Processing Team (DPT) to generate DTMs and DSMs. All LiDAR data used by the project are being collected by the Phil-LiDAR 1 Data Acquisition Component of the University of the Philippines Diliman. Validation and river bathymetry data gathered and provided to the project by Phil-LIDAR 1 Data Validation and Bathymetry Component of the UP Diliman are also processed by the DPT to assess accuracy of the LiDAR products and to incorporate river bed data into the DTMs. The DPT also extracts from the DSMs features such as houses, buildings, roads and bridges that will be utilized for mapping structures at risk to flooding.

The Flood Modeling Team (FMT) uses hydrologic and hydraulic simulation models such as the Hydrologic Engineering Center Hydrologic Modeling System and River Analysis System (HEC HMS and HEC RAS) to predict flooding due to rainfall of varying volume and intensity. The FMT uses the DTM and information extracted from the DSM during the flood modeling process. The main outputs of the FMT are flood models and flood hazard maps. The modeled and mapped flood hazards include historical (e.g., flooding due to Tropical Storms Agaton and Seniang) and hypothetical flood events. Hypothetical events are those flooding due to extreme rainfall events with return periods of 2-, 5-, 10-, 25-, 50-, and 25-year. The maps depict flood hazards into three levels: low (for flood depths of less than $0.50 \mathrm{~m}$ ), medium (for depths from $0.50 \mathrm{~m}$ to $1.50 \mathrm{~m}$ ), and high (for depths of greater than $1.50 \mathrm{~m}$ ). The FMT also incorporates all the information from the field surveys to assess whether the historical flood hazard maps generated are realistic and accurate or not. The flow measurement datasets are being used to calibrate and validate the upstream hydrological model to ensure that its computations of flow during rainfall events are accurate within acceptable levels of accuracy. The FMT together with the FST also conducts validation surveys to check the accuracy of the flood hazard maps. The teams visit random locations within a river basin and interview the nearest household about the occurrence of flooding in their locality. Information gathered during the surveys are the geographic locations of the households, the maximum depth of flood, the estimated dates when the flooding occurred, and the causes of flooding (if it was due to overflowing of the river, or an accumulation of rainwater).

\section{JABONGA AS A FLOOD-PRONE MUNICIPALITY AND CSU PHIL-LIDAR 1'S ATTEMPTS TO ASSESS THE PERENNIAL PROBLEM}

Jabonga is part of the Mainit-Tubay River Basin (Figure 1), and is one of the four municipalities located in the shores of Lake Mainit, considered as the Philippines' deepest and fourth largest lake. In fact, the municipality lies near the outlet of the lake.
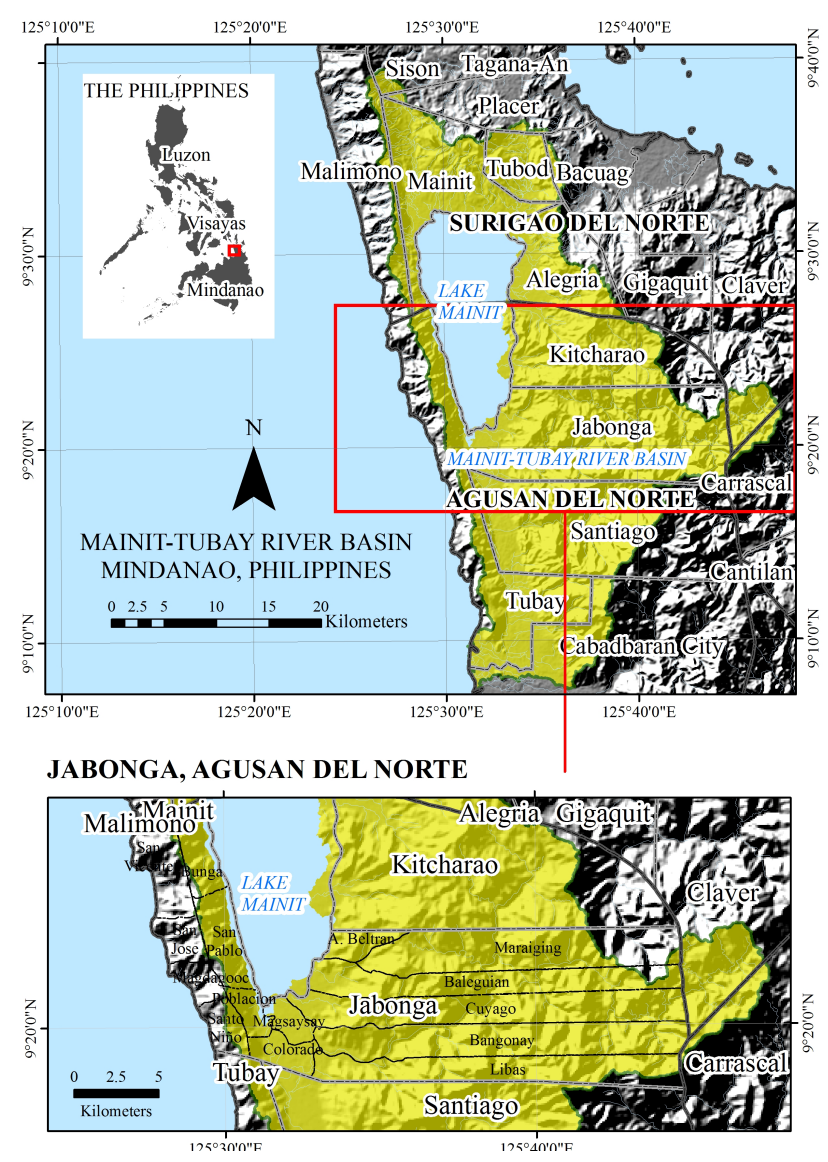

Figure 1: Series of maps showing the location of Jabonga municipality within the Mainit-Tubay River Basin in Mindanao, Philippines.

Lake Mainit receives inflows from several major and minor tributaries that drains upstream watersheds with a combined surface area of $870 \mathrm{~km}^{2}$ (LMDA, 2014). Every year, during the peak of the rainy season in the months of December and January (and sometimes until February), inflows from these tributaries increase the lakes water level and causes flooding of barangays within the municipalities located near the shore. One of these heavily affected municipalities is Jabonga. In February 2012, some 2,500 families in Jabonga were displaced by floods brought by a low pressure area that hit parts of Mindanao; a state of calamity was immediately declared in the municipality following an almost nonstop rainfall (Mascarinas, 2012). In January 2014, tropical storm Agaton (International name: Lingling) brought considerable volume of rainfall over several days that caused the lake to overflow, and in turn caused extensive flooding in the lakeshore barangays (NDRRMC, 2014; Crismundo, 2014). The flooding situation caused massive evacuation and discontinuity in land transportation due to flooded national highways near the lake (Crismundo, 2014). While flooding is a perennial problem in Jabonga, detailed flood hazard assessment was lacking at that time. 
As one of CSU Phil-LiDAR 1's project areas, flood hazard maps of Jabonga and the whole of Mainit-Tubay River Basin were prepared in 2015 according to the methodology explained in the previous section. These flood hazard maps depict the maximum depth and extent of flooding due to actual (or historical), e.g., Agaton (Figure 2) and Seniang (Figure 3) and hypothetical extreme rainfall events (e.g., rain return periods of 2, 5, 10, 25, 50, and 100 years). These maps have accuracies ranging from 68 $72 \%$ based on validation surveys conducted throughout the river basin (Amora et al., 2015).

A preliminary flood assessment using these hazard maps show that indeed Jabonga is very prone to flooding. Of its 15 barangays, 10 appear to be directly affected by flooding regardless of whether those flood events are actual or hypothetical ones. A simple spatial overlay analysis of the flood hazard maps with the municipal boundary would show that for the Agaton flood event, $0.5 \%$ of Jabonga's land surface area of approx. $270 \mathrm{~km}^{2}$ are under high hazard, $1.2 \%$ in medium hazard and $6.2 \%$ in low hazard. Combining these hazard levels makes $7.9 \%$ of Jabonga to be flooded. Although these statistics appears to be insignificant because the flooded areas are small compared to the total land surface area of Jabonga, it is still important considering that it is in these flooded areas where majority of the people are living. Two barangays, namely Magsaysay and Colorado, are the most affected by flooding, an important finding fully supported by news accounts (e.g., Mascarinas, 2012; NDRRMC, 2014; Crismundo, 2014). On the other hand, the hypothetical flood hazard maps reveals an increase in flood affected areas as the rain return period increase (i.e., as rainfall events becoming more extreme).

These flood hazard maps that the project generated for Jabonga municipality resulted from a river-basin wide flood modelling approach utilizing recent and detailed geospatial datasets and numerical models. Due to its fine detail, the maps allow deeper assessment of flooding such as identification of infrastructures (e.g., buildings/residences, roads and bridges) at risk of being flooded under various scenarios, finding areas that can be used as evacuation centers, identification and improvement of evacuation routes, and building flood defenses (specifically in areas where overflowing occurs), among many others . These hazard maps can also be used as vital input for localized land-use planning integrating flood hazard information.

\section{FORGING THE PARTNERSHIP WITH THE JABONGA LGU}

The opportunity for the hazard maps to be distributed and made useful to the Jabonga LGU came in May 28, 2015 when the Caraga Regional Office of the Philippines' Department of Science and Technology (DOST Caraga Region) invited the CSU Phil-LiDAR 1 project to participate in the launching of the 'Smarter Community Empowerment through Science and Technology (CEST)' project in Jabonga. It was in this launching that the preliminary flood hazard maps were turned-over to the Jabonga municipal officials (Figure 4) (CSU Phil-LIDAR 1, 2015).

It was after the map turnover that communication between the CSU Phil-LiDAR 1 Project and the Jabonga LGU through its Municipal Disaster Risk Reduction and Management Office (MDRRMO) began to materialized until it reached a stage that the two entities have engaged in a thorough discussion on what other aspects the project can be of value to make flood disaster management in Jabonga a 'smarter' one.

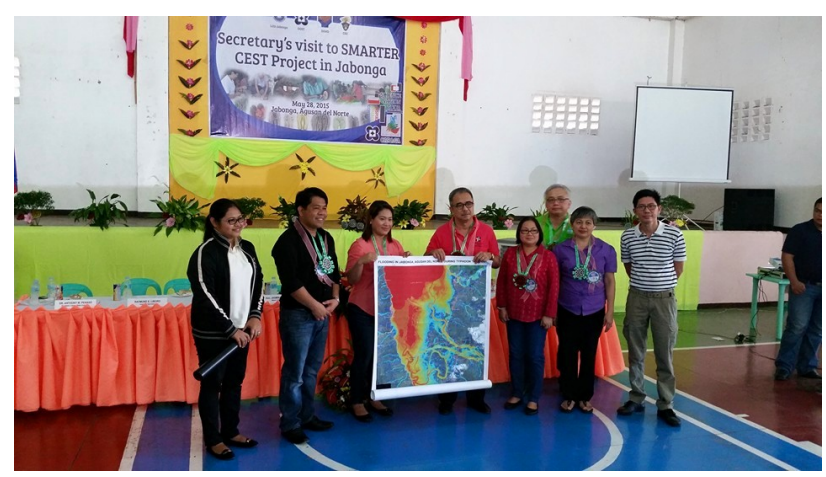

Figure 4: Flood hazard map turnover in the Jabonga municipality during the Smarter CEST Project launching which initiated the partnership between CSU Phil-LiDAR 1 Project and the Jabonga Local Government Unit.

The Jabonga MDRRMOs keen interest on having a 'smarter' approach in managing flood disasters by tapping the CSU PhilLiDAR 1 project to provide the approach and technology ended in a partnership in the development of an automated, near-real time web-based flood monitoring and assessment tool. The rationale for the partnership was anchored upon the MDRRMOs immediate need not only for timely and detailed flood hazard maps but also for easily-accessible geospatial tools and techniques that they can use before, during and after the occurrence of flood disasters. This smarter approach would mean the possibility of having timely, efficient and systematic dissemination of early warnings to communities; for determining before-hand sets of information necessary for careful planning and preparation of evacuation strategies; for easily identifying communities that need immediate action; in identifying areas that should be avoided; and in estimating the severity of damage to people and infrastructure as flooding progresses.

\section{FLOOD EVIDENS: A WEB-BASED APPLICATION FOR SMARTER FLOOD DISASTER MANAGEMENT IN JABONGA}

CSU Phil-LiDAR 1's response to make flood disaster management in Jabonga 'smarter' was through the development of the Near-real Time Flood Event Visualization and Damage Estimations (Flood EViDEns) customized web application. Flood EViDEns is actually a generic web application developed by the project which served as a visualization and analysis platform for all hazard maps generated by the project with a basic set of functionalities to conduct spatial overlay analysis of flood hazards and elements-at-risk (e.g., buildings). A public version of the generic application is available in http://evidens.csulidar1.info. Its development is discussed in detail in Santillan et al. (2015).

In the case of Jabonga, the very basic functionality of Flood EViDENs would not suffice the LGUs requirements of 'smarter' flood disaster management. As such, a customized version was developed utilizing LiDAR-derived elevation and information products as well as other elevation datasets, water level records by monitoring stations, flood simulation models, flood hazard maps, and socio-economic datasets (population, household information, etc.), in order to visualize in near-real time the current and future extent of flooding, to disseminate early warnings, and to provide maps and statistics of areas and communities affected and to be affected by flooding.

In this partnership, the CSU Phil-LiDAR 1 was tasked to develop the application, deploy it in the Jabonga LGU (using the MDR- 

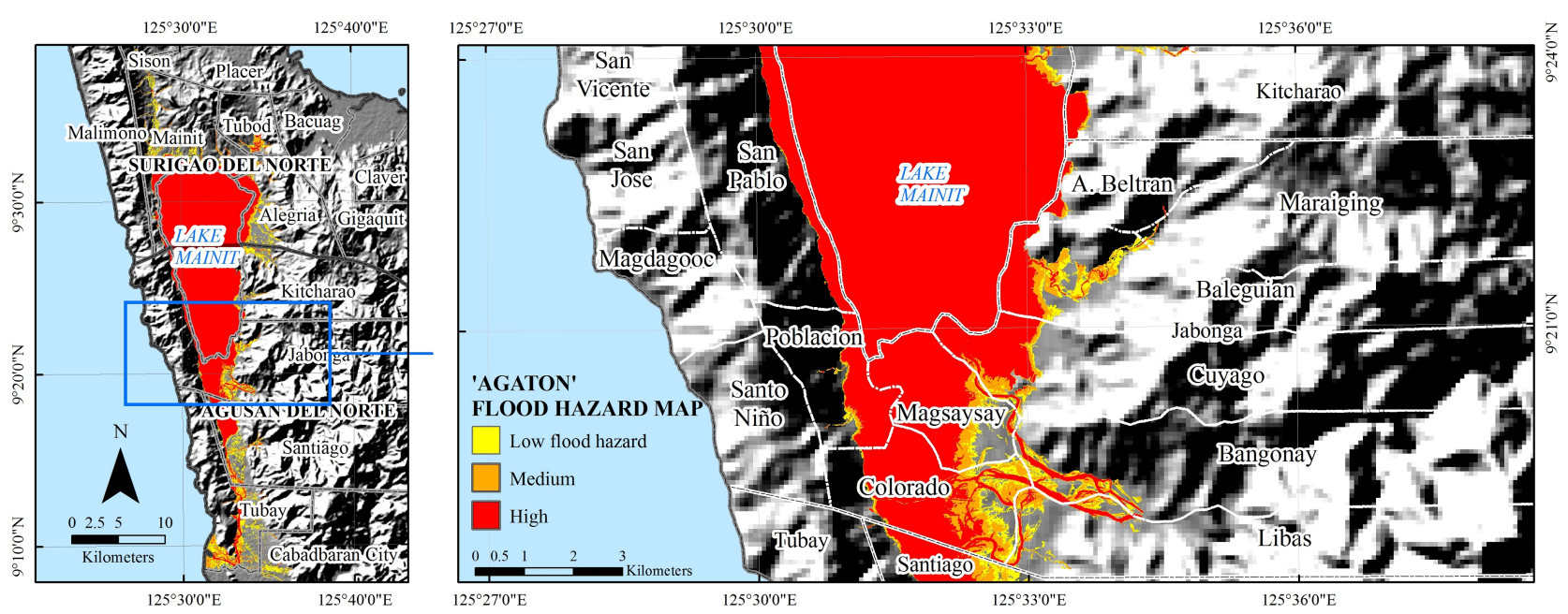

Figure 2: Flood hazard map of Jabonga for the Agaton event.
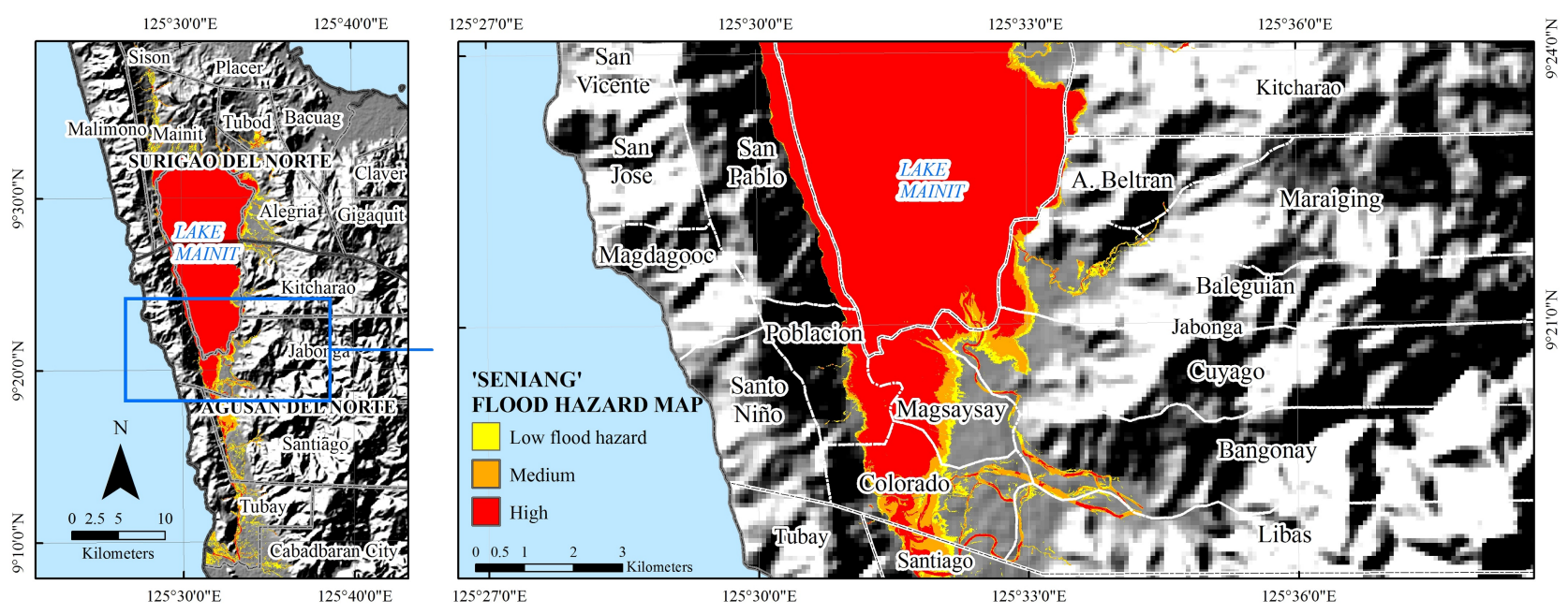

Figure 3: Flood hazard map of Jabonga for the Seniang event

RMOs hardware) and to train the Jabonga LGU on how to use the application, including capacity-building activities such as seminars and trainings on the use of geospatial technologies. On the other hand, the Jabonga LGU will provide household-level socioeconomic datasets (household information such as number of family members, sources of incomes, type of housing materials, etc.) that are crucial to generate the expected outputs of the web application.

So far, we have developed a preliminary but working version of Flood EViDENs customized for Jabonga. It is considered preliminary since the gathering of household level information by the MDRRMO is not yet finished, and is expected to be completed before the year 2016 ends. As a web application, it was configured to display maps of ood hazards and affected structures as well as processed textual information (e.g., statistics of affected structures) (Figure 5). It allows the user to display a summary of the number of structures affected by a ooding event, as well as a web map showing ood hazard, structures (buildings), and other associated layers (e.g., political boundaries). In the web page, a user can display both the near-real time ood hazard map as well as location of affected structures which are color-coded according to hazard level. Clicking in an affected structure in the web map will display important attributes such as name of structure (head of the family if the structure is a residential building), type, location, and many others. This feature was made possible using the rich dataset of household information collected by the Jabonga MDRRMO and provided to the project. The application also has a Search/Filter utility where the user can search ood affected structures according to barangay and type of structure. The resulting list categorizes the structures according to ood hazard level, and if clicked, the user can see the actual location of these structures in the web map (e.g., the structures location is zoomed-in in the map). In addition to these functionalities, plots/graphs showing the actual status of the lakes water level in the last 24 hours and forecast for the next 12 hours (as computed by the water level forecasting model) are also available in Flood EViDEns. This allows the user to monitor the lakes water level, and determine whether it will reach the lakes spilling level in the next hours, including the specic date and time of spilling.

Figure 6 illustrates the rich information that Jabonga LGU and communities can extract from Flood EViDEns when applied during times of flood disaster (e.g., during January 2014 flooding caused by tropical storm Agaton). In this example, the application was able to provide a summary of affected structures categorized into three hazard levels as well as the map of ooding that have occurred on January 20, 2014 11:10 PM, approximately one day after the impact of the tropical storm. Also shown in the map are the affected structures, color coded according to hazard level. 

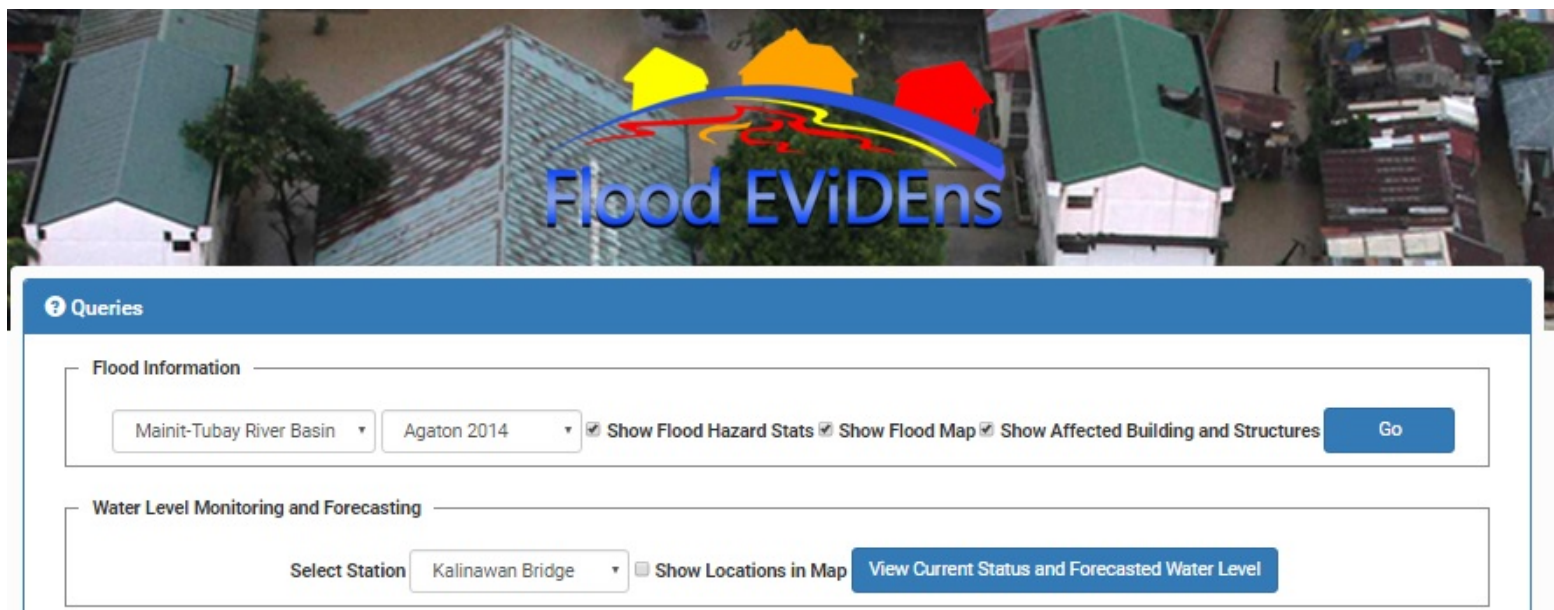

B) Flood Hazard Information
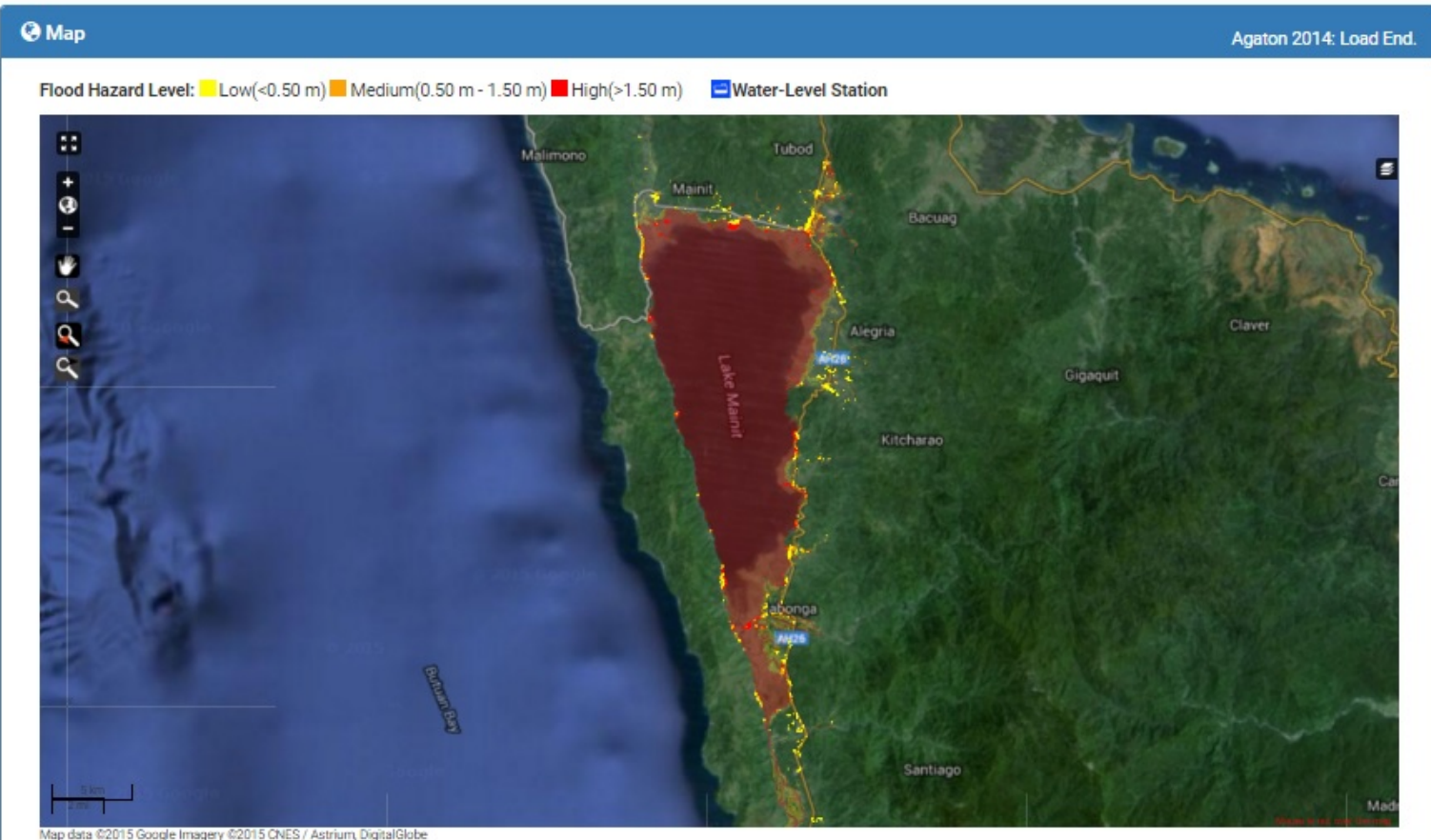

(i) Detailed Flood Hazard Information

Figure 5: Graphical user interface of Flood EViDEns.

Household information can also be viewed in addition to locating those affected structures (Figure 7).

\section{CONCLUDING REMARKS}

The partnership between the CSU Phil-LiDAR 1 project and the Jabonga LGU discussed in this paper can be considered an attempt to bring geospatial technologies to the local level. The development of Flood EViDEns as the main product of the partnership is an important application of geospatial technologies that will greatly improve localized flood disaster management in this municipality, e.g., by allowing smarter and geospatially-informed decision making before, during, and after a flood disaster.

\section{ACKNOWLEDGEMENTS}

This work is an output of the Caraga State University (CSU) PhilLiDAR 1 project under the "Phil-LiDAR 1. Hazard Mapping of the Philippines using LiDAR" program funded by the Department of Science and Technology (DOST). The LiDAR datasets used 


\section{(9) Flood Hazard Information}

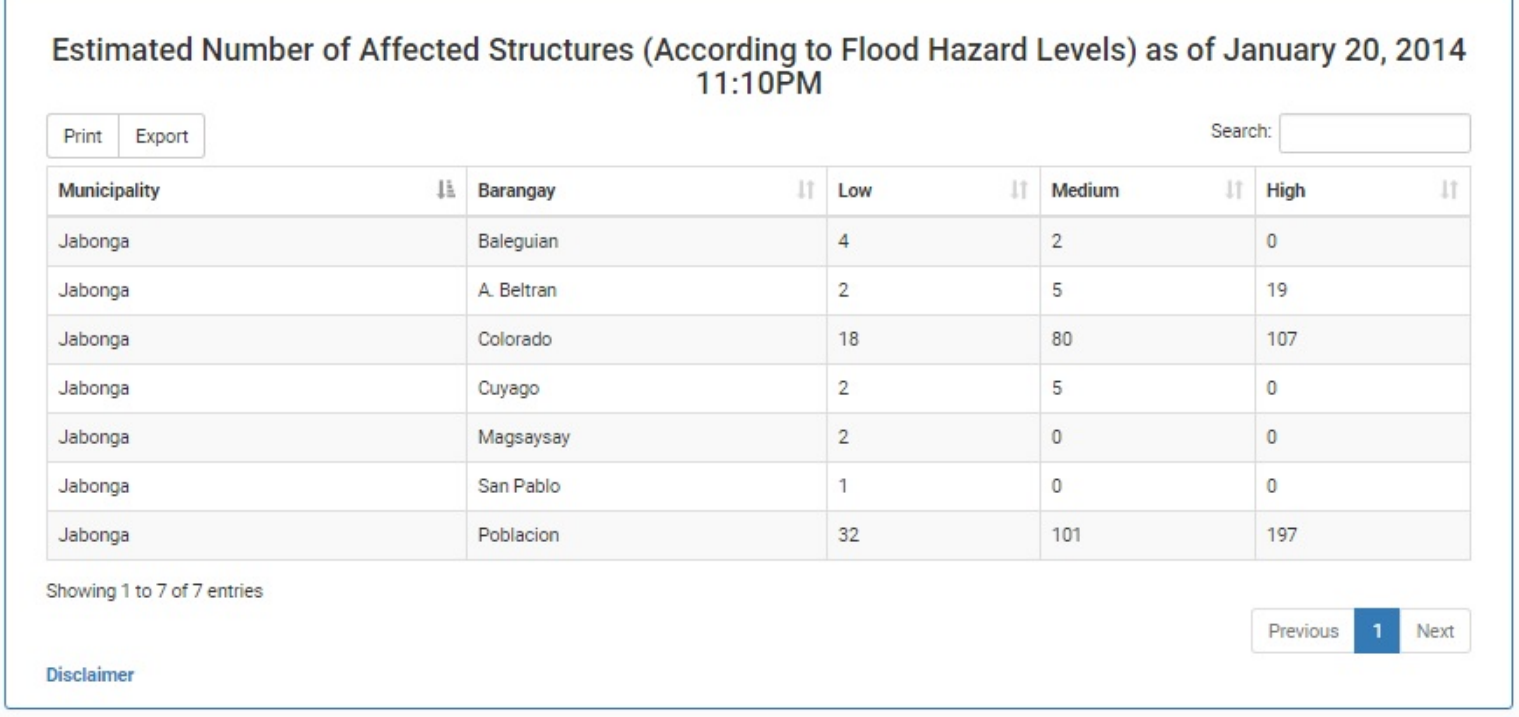

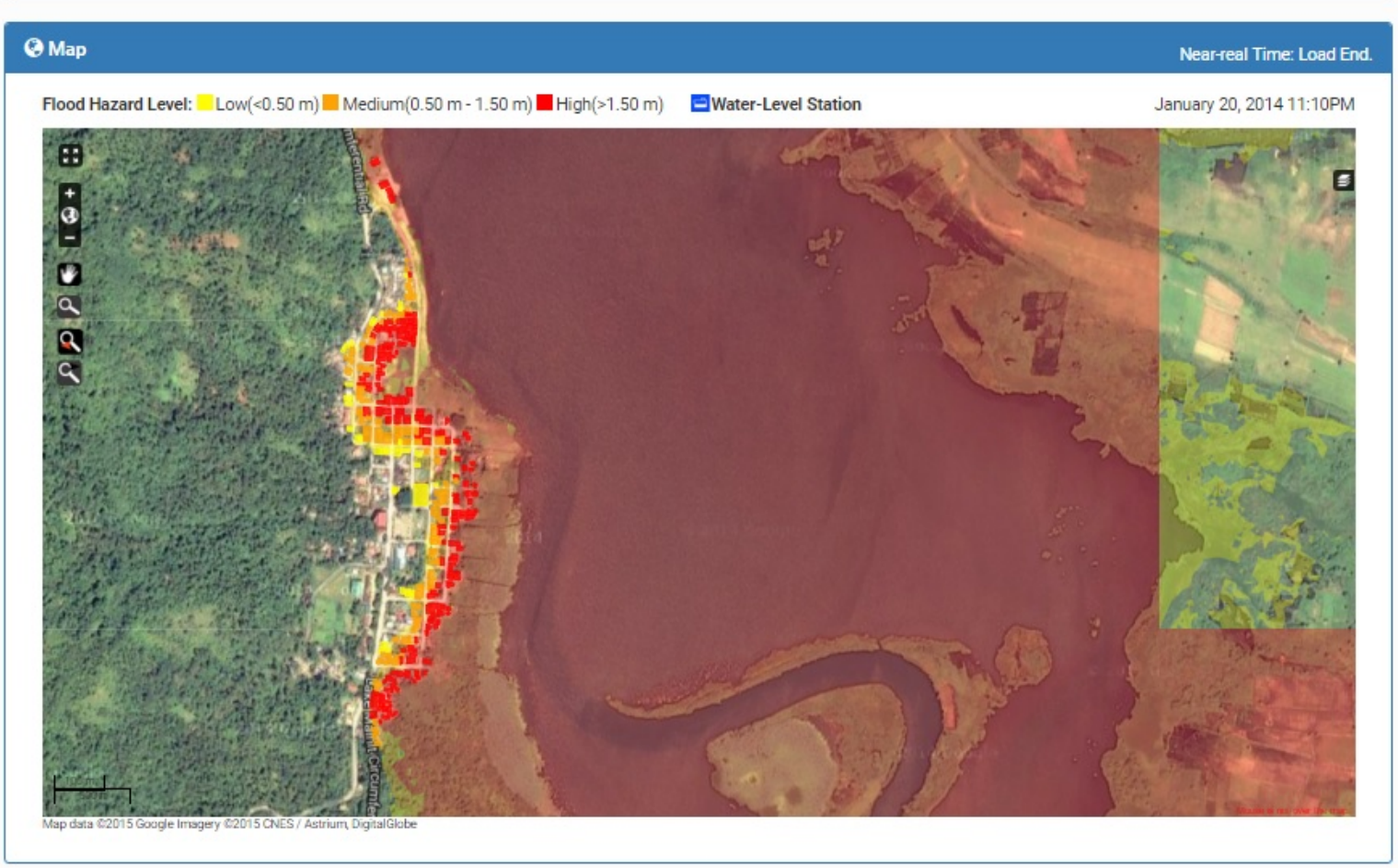

Figure 6: Applying Flood EVIDEns to generate statistics and map of ooding and affected structures.Flooded areas and affected structures are color-coded according to hazard level (yellow: low; orange: medium; red: high).

in this work were provided by the University of the Philippines Disaster Risk and Exposure for Mitigation (UP DREAM)/PhilLIDAR 1 Program.

We sincerely acknowledge the unending support of the Municipal Disaster Risk Reduction and Management Office (MDRRMO) of Jabonga, Agusal del Norte as well as the DOST Caraga Regional Office for making possible the forging of the partnership. The CSU Phil-LiDAR 2 Project is also acknowledged for allowing us to host in their dataserver the public version of Flood EViDEns.

We are thankful to the DOST and the Philippine Council for Industry, Energy and Emerging Technology Research and Development (PCIEERD) for the financial assistance that allowed us to present this paper during the XXIII ISPRS Congress 2016.

\section{REFERENCES}

Amora, A., Santillan, J.R., Makinano-Santillan, M., Marqueso, J.T., 2015. Flood hazard mapping of Mainit-Tubay River Basin, Mindanao, Philippines. In: 36th Asian Conference on Remote Sensing 2015 (ACRS 2015): Fostering Resilient Growth in Asia, Quezon City, Metro Manila, Philippines, Vol. 1, pp. 440-450.

Crismundo, M., 2014. Flood displaces thousands in Caraga. http://www.mb.com. ph/flood-displaces-thousands-in-caraga/ (30 April 2014).

CSU Phil-LiDAR 1, 2015. Ceremonial turnover of preliminary flood maps by DOST Secretary Montejo to Jabonga local government unit, http://csulidar1.info/news-and-events/ceremonial- 


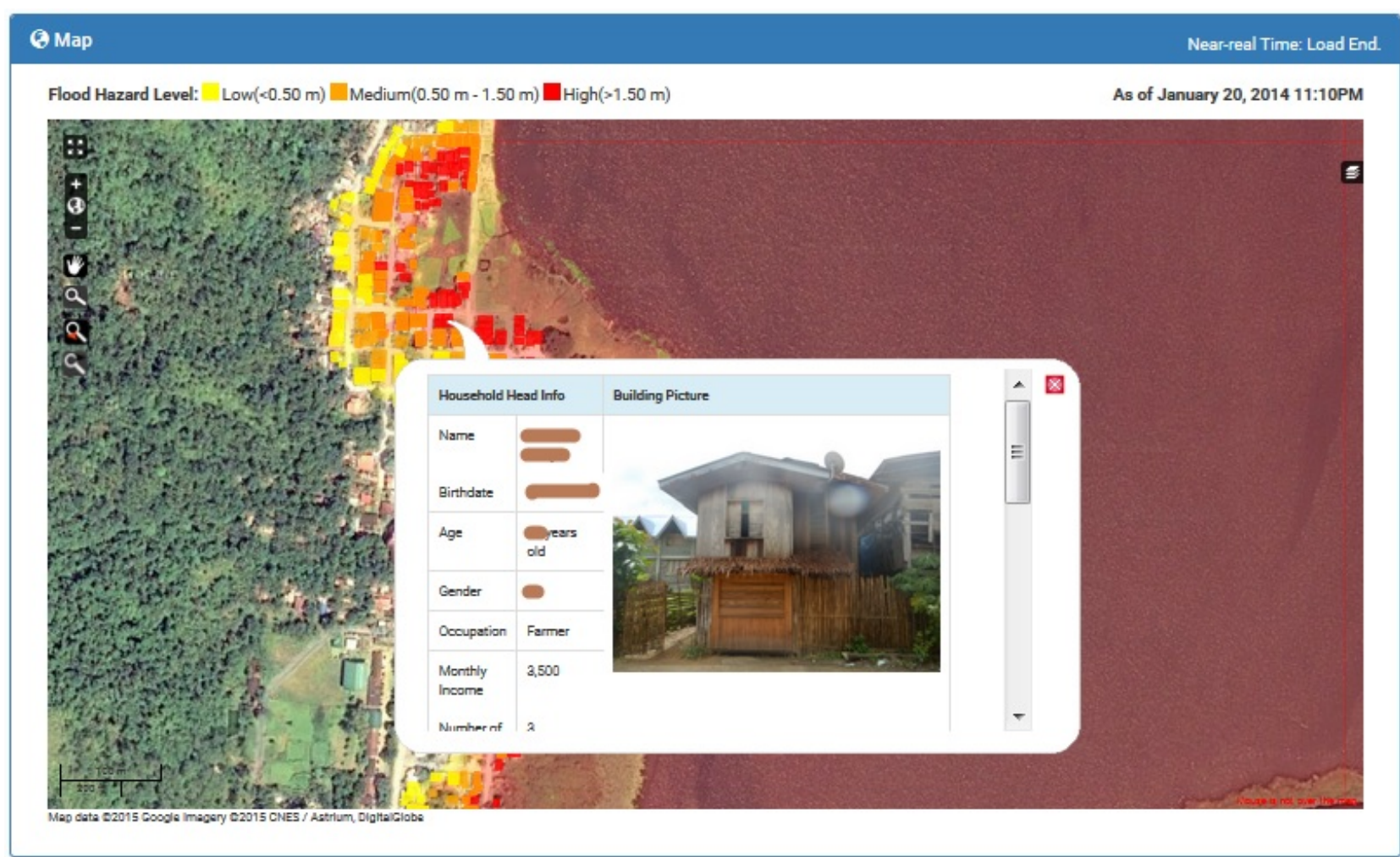

Figure 7: Viewing household-level information of an affected structure such as this is another feature of Flood EViDENs.

turnover-of-preliminary-flood-maps-by-dost-secretary-montejoto-jabonga-local-government-unit/ (01 February 2016).

DRRM-CEP, 2014. National Disaster Response Plan as of June 2014. Disaster Risk Reduction and Management Capacity Enhancement Poject (DRRM-CEP) of the Japan International Cooperation Agency (JICA) for the Office of Civil Defense (OCD), Government of the Republic of the Philippines. http://www.ndrrmc.gov.ph/attachments/article/1334/NDRP _Hydro_Meteorological_Hazards_as_of_2014.pdf (05 January 2016).

LMDA, 2014. Lake Mainit Development Alliance (LMDA) Website, "Physical Features of Lake Mainit", https://lmda.wordpress.com/about-lake-mainit/physical-featureof-lake/ (30 April 2014).

Makinano-Santillan, M., Santillan, J.R., 2015. Flood hazard mapping of river basins in Caraga Region, Mindanao, Philippines through the CSU Phil-LiDAR 1 project. In: 36th Asian Conference on Remote Sensing 2015 (ACRS 2015): Fostering Resilient Growth in Asia, Quezon City, Metro Manila, Philippines, Vol. 1, pp. 599-610.

Mascarinas, E., 2012. 2,500 families displaced by floods in Agusan. MindaNews, http://www.mindanews.com/environment/floods/2012/02/18/2500families-displaced-by-floods-in-agusan/ (30 April 2014).

NDRMMC, 2011. National Disaster Risk Reduction and Management Plan (NDRRMP) 2011-2028. National Disaster Risk Reduction and Management Council (NDRRMC), http://www.ndrrmc.gov.ph/attachments/article/41/NDRRM

_Plan_2011-2028.pdf (05 January 2016).

Santillan, J.R., Morales, E.M.O, Makinano-Santillan, M., 2015. Flood EViDEns: a web-based application for near-real time ood event visualization and damage estimations. In: 36th Asian Conference on Remote Sensing 2015 (ACRS 2015): Fostering Resilient Growth in Asia, Quezon City, Metro Manila, Philippines, Vol. 4, pp. 2655-2664. 